\title{
Alteration of F-waves by Motor Imagery With and Without Hitting in Badminton \\ Yujiro Masu ${ }^{1, *}$, Ryo Udaka ${ }^{2}$ and Ken Muramatsu ${ }^{3}$
}

${ }^{1}$ Department of Physical Therapy, Health Science University, Yamanashi, Japan

${ }^{2}$ Rehabilitation unit, Yokohama Asahi Chuo General Hospital, Kanagawa, Japan

${ }^{3}$ Department of Physical Therapy, Kyorin University, Tokyo, Japan

\section{Abstract}

Background: In this study, therefore, we directed our attention to motion images of badminton and evaluated the effects of the presence or absence of actual hitting of the shuttle on $\mathrm{F}$ waves from the short abductor muscle of the thumb to collect information about the excitability of spinal motor neurons.

Methods: The subjects were 10 male and female college students with no experience in playing badminton. F waves were recorded under 3 different conditions: 1) having the subject raise their arm without holding the racket and take a posture ready to return the flying shuttle by imagining the motion (non-racket posture; NRP), 2) having the subject hold the racket and take a posture ready to return the flying shuttle by imagining the movement (racket posture; RP), and 3) having the subject actually keep rallies for 2 minutes and, then take a posture to return the flying shuttle by imagining the movement (stroke posture; SP) .

Results: The amplitude of $\mathrm{F}$ waves relative to the maximum $\mathrm{M}$ wave was significantly larger in $\mathrm{RP}$ and $\mathrm{SP}$ than in NRP. The minimum latency of the F waves was significantly shorter in SP than in NRP.

Conclusion: The results suggest that the excitability of spinal motor neurons is suppressed by holding the racket and that the conduction velocity of excitation in muscle fibers is increased by actually performing strokes.

\section{Introduction}

Research of mental representation of physical activities in the brain has shown motor areas centering on the primary motor area involved in an action can be activated simply by imagining the action even without actually performing it $[1,2]$. However, in people who have not experienced the action, an adequate image of the action cannot be formed by imagining, and the motor areas are not activated [3]. Ting L et al. [4] carried out an experiment in which they had novice subjects practice badminton for 12 weeks, and then had them expect the trajectory of a shuttle by viewing videos. By measuring brain waves during this experiment, the generation of $\mathrm{N} 2$ and $\mathrm{P} 3$ components larger than the event-related potential was confirmed in the practice group, indicating an improvement in the ability to expect shuttle movement compared with the control group. From these results, badminton players are considered to observe the motion pattern of the opponent, simulate the motion in the brain, and expect where the shuttle is aimed to be hit. Moreover, the results suggest that excitability of spinal motor neurons in receiving is suppressed by continuing to play badminton [5] and that a more rapid swing of the racket becomes possible by instantaneous change of muscle activities of the lower extremities associated with posture control and instantaneous synchronized mobilization of a large number of motor units [6]. Thus, by suppressing the activity of the myotatic reflex arc during receiving, badminton players are considered to reduce the effects of spinal reflex, which affect motion, and facilitate the execution of motions faithfully reproducing motor commands from the cerebrum.

$\mathrm{F}$ and $\mathrm{H}$ waves observed by applying electric stimulation to muscles are used in methods to evaluate the excitability of spinal motor neurons [7-11]. An F wave is an evoked electromyographic signal resulting from retrograde excitation of high-threshold a motor neurons, which are not excited by excitatory synaptic inputs from Ia afferent nerve fibers, by retrograde action potentials generated a motor neurons, which are not excited by excitatory synaptic inputs from Ia afferent nerve fibers, by retrograde action potentials generated by maximum electric stimulation of peripheral nerve bundles. An $\mathrm{H}$ wave, on the other hand, is an evoked electromyographic signal resulting from monosynaptic excitation of a motor neurons by Ia afferent nerve fibers excited by electric stimulation of peripheral nerve bundles. Both are indices for the assessment of the excitability of spinal motor neurons. However, stable recording to $\mathrm{H}$ waves is reportedly difficult at sites other than the triceps muscle of the calf, and $\mathrm{F}$ waves, which can be recorded consistently, are used to evaluate the excitability of motor neurons that supply the upper extremities. $F$ waves can be detected from all muscles innervated by the spinal nerves and are advantageous in that they can be easily recorded in various body positions, especially because they are recorded by applying a very strong electric stimulation, i.e., twice the strength of stimulation at which the maximum $\mathrm{M}$ wave is detected, while $\mathrm{H}$ waves are recorded by applying very mild electric stimulation to the subjects. By utilizing the above characteristics of $\mathrm{F}$ waves, it may be possible to evaluate the characteristics of the excitability of spinal motor neurons involved in the arm movements before a badminton player swings the racket and to answer the question of why badminton players can swing the racket by instantaneously responding to the flying shuttle.

In this study, therefore, we directed our attention to motion images of badminton and evaluated the effects of the presence or absence

"Corresponding Author: Dr. Yujiro Masu, Department of Physical Therapy Health Science University, 7187 Kodachi, Fujikawaguchiko, Yamanashi 401- 0380, Japan; Tel: +81 55583 5298; E-mail: y-masu@kenkoudai.ac.jp

Citation: Masu Y, Udaka R, Muramatsu K (2019) Alteration of F waves by Motor Imagery With and Without Hitting in Badminton. Int J Phys Ther Rehab 5: 158. doi: https://doi.org/10.15344/2455-7498/2019/158

Copyright: (C) 2019 Masu et al. This is an open-access article distributed under the terms of the Creative Commons Attribution License, which permits unrestricted use, distribution, and reproduction in any medium, provided the original author and source are credited. 
Citation: Masu Y, Udaka R, Muramatsu K (2019) Alteration of F waves by Motor Imagery With and Without Hitting in Badminton. Int J Phys Ther Rehab 5: 158. doi: https://doi.org/10.15344/2455-7498/2019/158

Page 2 of 5

of actual hitting of the shuttle on $\mathrm{F}$ waves from the short abductor muscle of the thumb to collect information about the excitability of spinal motor neurons.

\section{Methods}

\section{Subjects}

The subjects were 10 male and female college students with no experience in playing badminton (age: $20.5 \pm 1.2$ years, height: $167.5 \pm 9.8 \mathrm{~cm}$, body weight: $60.3 \pm 13.2 \mathrm{~kg}$, all right-handed). The objective and safety of the experiment were explained to all subjects, and their voluntary consent to participate in the experiment was obtained. This study was carried out with approval by the Research Ethics Committee of Health Science University.

\section{Method for the measurement of $F$ waves}

F waves were measured using Neoropack (NIHON KOHDEN). A silver plate recording electrode was attached to the skin on the short abductor muscle of the thumb of the racket-holding hand, and a bipolar stimulation electrode was placed on the wrist (Figure 1). Electric stimulation was applied percutaneously to the median nerve, and $\mathrm{M}$ and $\mathrm{F}$ waves from the short abductor muscle of the thumb were recorded. The strength of stimulation was twice the level at which the maximum $\mathrm{M}$ wave was observed, the stimulation frequency was 0.2 $\mathrm{Hz}$, and the number of stimulations was $20 . \mathrm{F}$ waves were recorded under 3 different conditions (Figure 2): 1) having the subject raise their arm without holding the racket and take a posture ready to return the flying shuttle by imagining the motion (non-racket posture; NRP), 2) having the subject hold the racket and take a posture ready to return the flying shuttle by imagining the movement (racket posture; RP), and 3) having the subject actually keep rallies for 2 minutes and, then take a posture to return the flying shuttle by imagining the movement (stroke posture; SP) (Figure 3). The order of measurements under the 3 conditions was individually arranged at random.

From the amplitudes observed in each posture, $\mathrm{F}$ wave was standardized as the percentage of $\mathrm{M}$ waves at maximum stimulation, and the largest amplitude in 20 trials was adopted as $\mathrm{F}$ wave. Regarding the latency of $\mathrm{F}$ waves, the shortest in 20 measurements was adopted (Figure 4).

Inter-group comparisons were made by one-way analysis of variance, and, if a significant main effect was observed in the factors, the Tukey-Kramer multiple comparison test was performed. The level of significance was $\mathrm{p}<0.05$.

\section{Results}

Figure 5 shows the amplitude of $\mathrm{F}$ waves relative to the maximum $M$ wave. The value was significantly larger in RP and SP than in NRP $(\mathrm{p}<0.05)$.

Figure 6 shows the minimum latency of the $\mathrm{F}$ waves. It was significantly shorter in SP than in NRP $(\mathrm{p}<0.05)$.

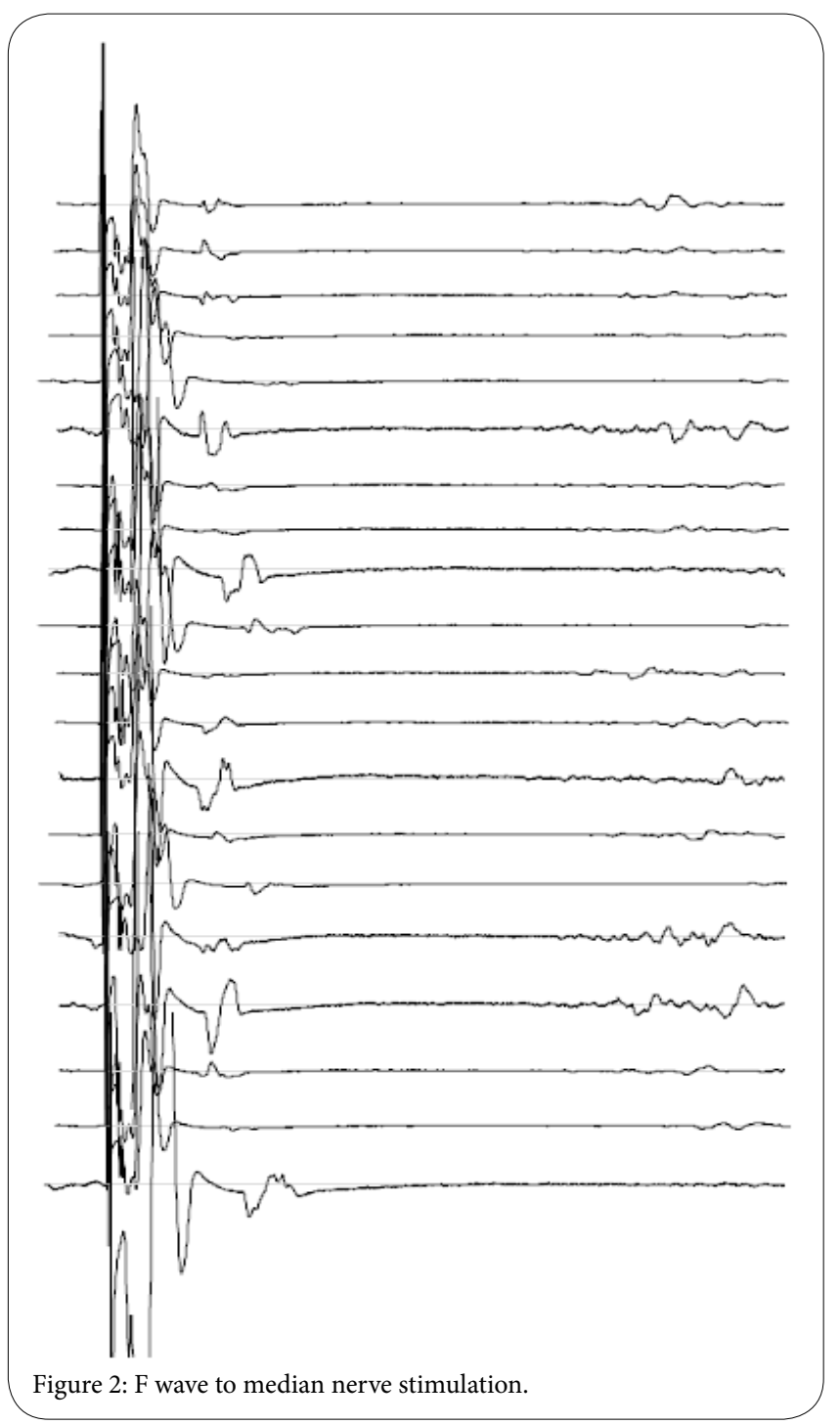

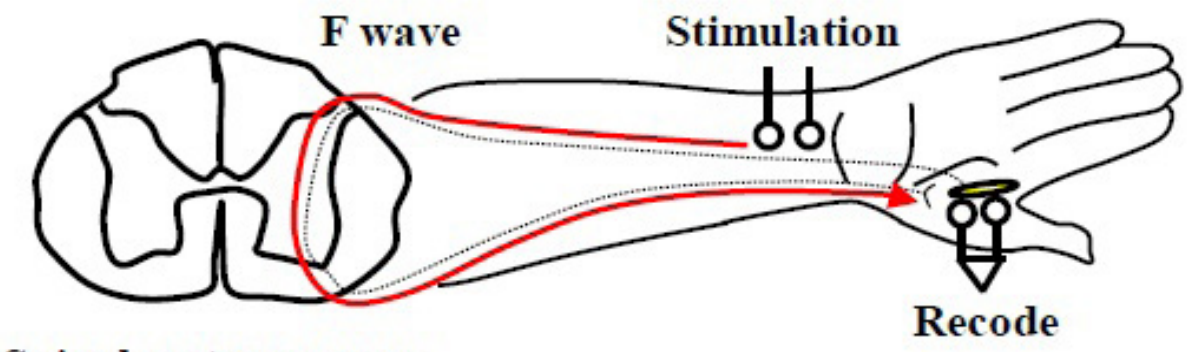

\section{Spinal motor neuron}

Figure 1: Schematic drawing of experimental setup. 


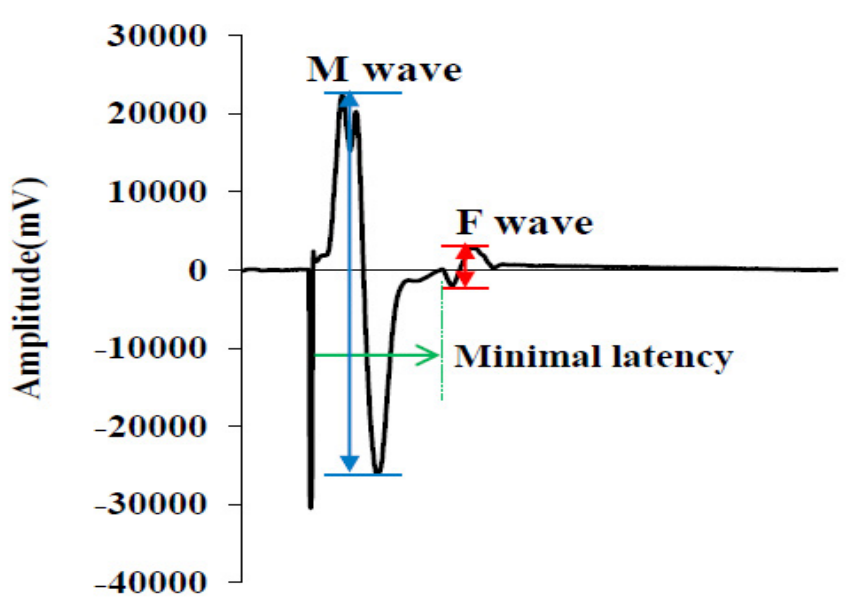

Figure 3. Definition of $\mathrm{M}$ wave, $\mathrm{F}$ wave and minimal latency.

( 1 )

No-stroke

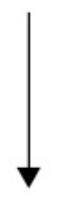

Only hand

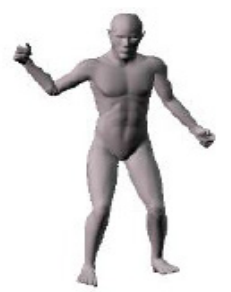

( 2 )

No-stroke

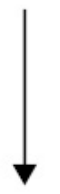

Grip a racket

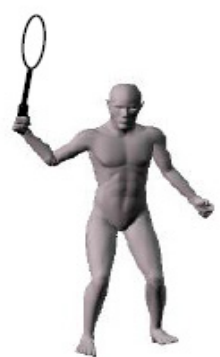

( 3 )

Rally for 2 minutes

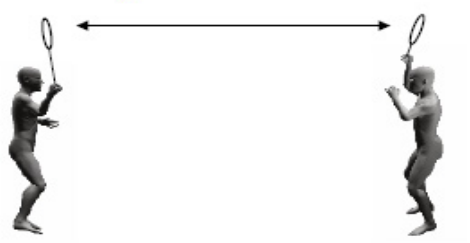

Grip a racket

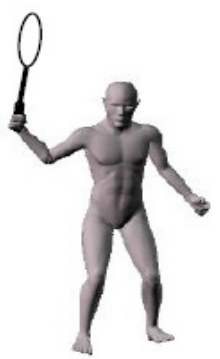

Figure 4. Schematic drawing of each measurement condition.

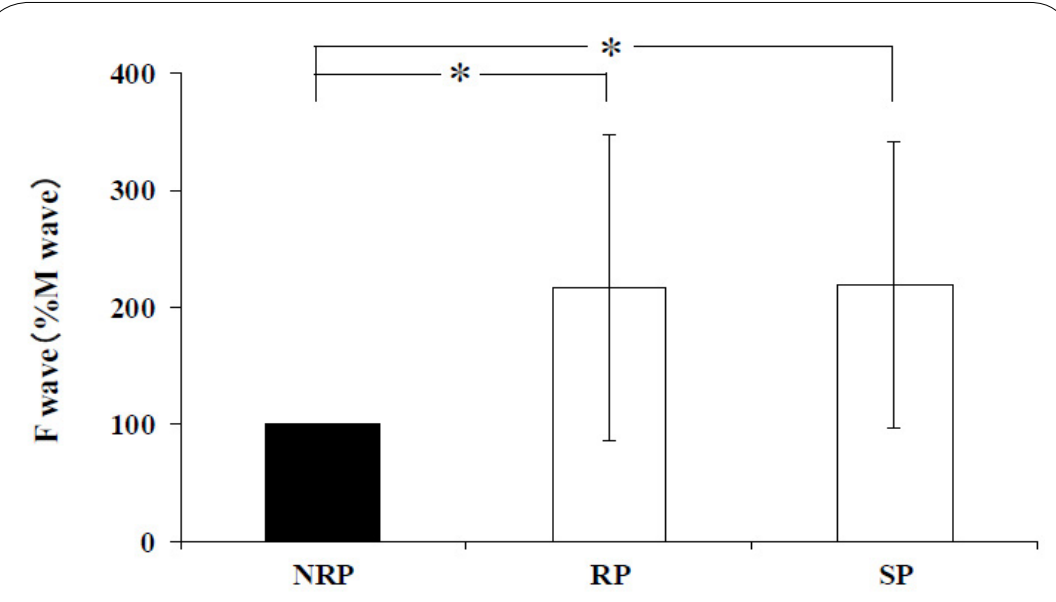

Figure 5: Comparisons of F wave in NRP, RP and SP.

The F-wave rate of each condition was calculated, using the values when NRP as $100 \%$.

The error bars indicate the standard error of the mean. Significant difference ${ }^{\star} p<0.05$ 


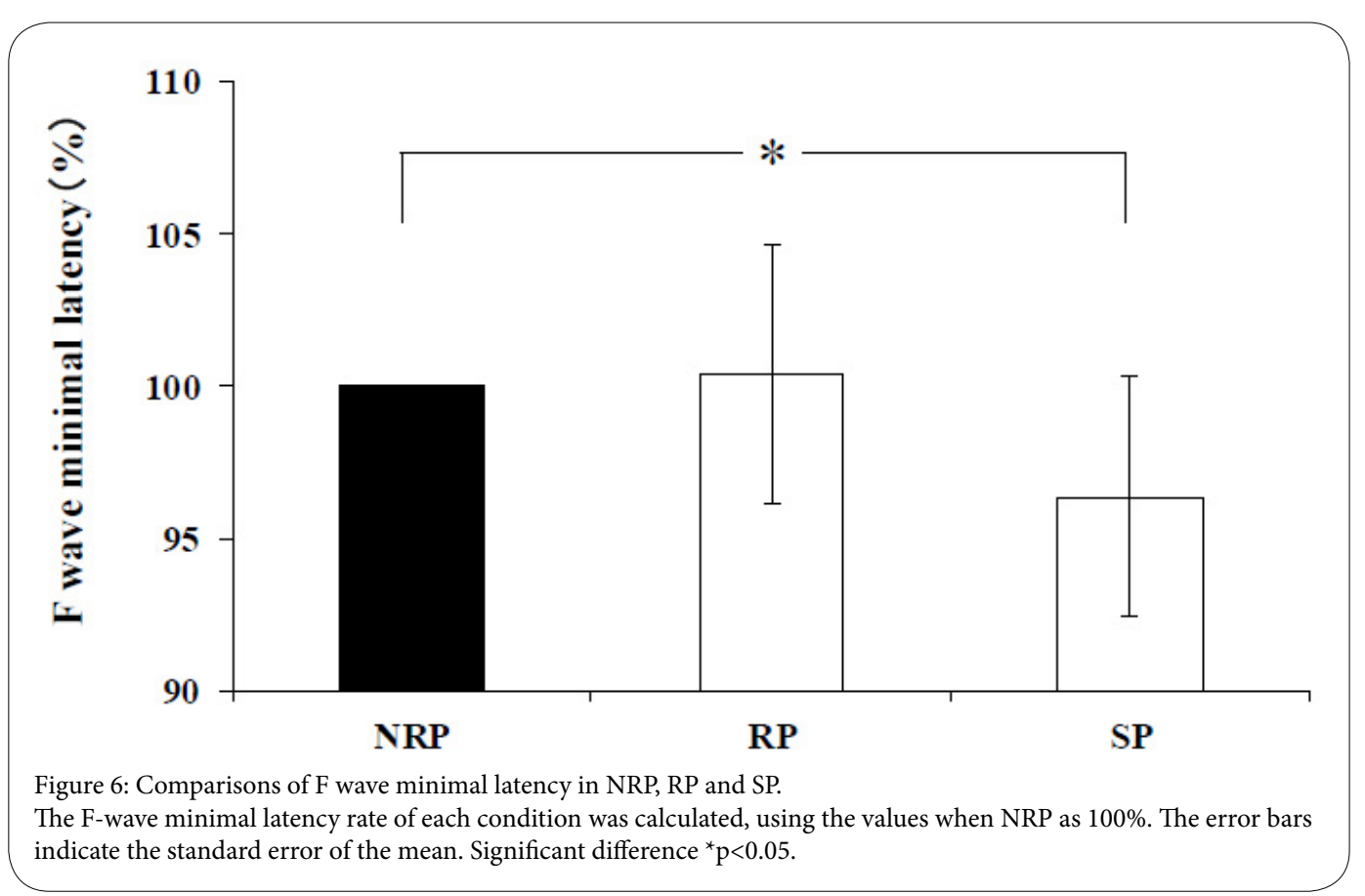

\section{Discussion}

F waves are considered to be caused by retrograde excitation of large high-threshold motor neurons that are not excited by excitatory synaptic inputs from Ia afferent fibers when peripheral nerves are electrically stimulated at the maximum level. Since the amplitude of an $\mathrm{F}$ wave is considered to reflect the number of motor neurons the firing threshold of which is not exceeded by excitatory synaptic inputs from Ia afferent fibers, the amplitude of an $\mathrm{F}$ wave is larger as the spinal excitability is lower. In this study, the amplitude of the F waves was significantly larger in RP and SP than in NRP. Therefore, the spinal excitability is considered to have been lower in RP and SP than in NRP. The functional significance of the reduction in spinal excitability in RP and SP is unclear. However, high spinal excitability is considered to be advantageous for exciting a large number of motor neurons, because the membrane potential of motor neurons is elevated, but to make movements precisely controlled by the brain difficult, because they are more likely to be affected by spinal reflex. In contrast, if spinal excitability is maintained at a moderately reduced level, the membrane potential of motor neurons is considered to be lowered, the effect of spinal reflex to be reduced, and the execution of movements faithful to directions by the brain to be facilitated.

According to studies of event-dependent changes in spinal excitability using $\mathrm{H}$ reflex of the soleus muscle as an index, the excitability of spinal motor neurons was more likely to be increased in experienced than non-experienced swimmers [13] but tended to be suppressed in professional ballet dancers [14], probably because swimmers are required to exert explosive muscle contraction in competition but ballet dancers are more expected to perform movements that faithfully reproduce motor directions from the cerebrum. There has also been a report that long-term training using a particular tool leads to functional remodeling of the motion cortical network in athletes and that the motor areas are more excited in motion imaging, holding than not holding the badminton racket [15] These observations suggest that holding the racket activates the motor areas and suppresses myotatic reflex. Moreover, in this study, the latency of the $\mathrm{F}$ waves was significantly shorter in SP than in NRP. This suggests that the muscle temperature was elevated, and the muscle blood flow was increased, by performing the stroke movement, leading to an improvement in ATP production and shortening of the latency. Shortening of the latency of $\mathrm{F}$ waves means increased conduction velocity of excitation, which is considered to contribute to improved motor characteristics of muscle function. The improvement in motion skills is promoted by motion imaging after practicing physical movements [16,17]. Although keeping rallies for 2 minutes was not sufficient to suppress the excitability of spinal motor neurons in this study (no significant difference between RP and SP), actually having rallies followed by motion imaging is considered to be more effective for improving performance.

Repeated imagining of movements aiming to improve the motion skill (motor imagery) has a beneficial effect as perceptive training and improves the performance [18]. Particularly, motor imagery with attention to muscular sensation is important for improving the motion skill [19]. However, there is a gap between the clarity of subjective motor imagery and precision of execution of imagined motion, and they have been reported not to be always correlated [20]. Particularly, there are individual differences in the ability of motor imagery, and it is difficult to objectively assess one's motor imagery by oneself. Therefore, the excitability of spinal motor neurons, which is likely to be affected by motor imagery, is considered useful as an index for the evaluation of the accuracy of motor imagery.

\section{Conclusion}

In this study, focusing on motor imagery of badminton, we evaluated the effects of the presence or absence of actual hitting of the shuttle on $\mathrm{F}$ waves of the short abductor muscle of the thumb. The results suggest that the excitability of spinal motor neurons is suppressed by holding the racket and that the conduction velocity of excitation in muscle fibers is increased by actually performing strokes. 
Citation: Masu Y, Udaka R, Muramatsu K (2019) Alteration of F waves by Motor Imagery With and Without Hitting in Badminton. Int J Phys Ther Rehab 5: 158. doi: https://doi.org/10.15344/2455-7498/2019/158

\section{Funding}

This work was supported by a Research Grant of the Health Science University.

\section{Competing Interests}

The authors declare that they have no competing interests.

\section{References}

1. Ehrsson HH, Geyer S, Naito E (2003) Imagery of voluntary movement of fingers, toes, and tongue activates corresponding body-part-specific motor representations. J Neurophysiol 90: 3304-3316.

2. Naito E, Nakashima T, Kito T, Aramaki Y, Okada T, et al. (2007) Human limbspecific and non-limb-specific brain representations during kinesthetic illusory movements of the upper and lower extremities. Eur J Neurosci 25 : 3476-3487.

3. Mulder T, Zijlstra S, Zijlstra W, Hochstenbach J (2004) The role of motor imagery in learning a totally novel movement. Exp Brain Res 154: 211-217.

4. Ting L, Mengling S, Desheng Y, Yongjie L, Nan Y, et al. (2017) The effect of badminton training on the ability of same-domain action anticipation for adult novices: Evidence from behavior and ERPs. Neurosci Lett 660: 6-11.

5. Masu Y, Muramatsu K (2015) Soleus H-reflex modulation during receive stance in badminton players in the receive stance. J Phys Ther Sci 27: 123125.

6. Masu Y, Nagai M (2016) Characteristics of lower limb muscle activity during upper limb elevation in badminton players. J Phys Ther Sci 28: 2510-2514.

7. Ibrahim IK, el-Abd MA (1997) Giant repeater F-wave in patients with anterior horn cell disorders. Role of motor unit size. Am J phys Med Rehabi 76: 281-287.

8. Wallbom AS, Geisser ME, Haig AJ, Koch J, Guido C, et al. (2008) Alterations of Fwave parameters after exercise in symptomatic lumber spinal stenosis. Am J phys Med Rehabil 87: 270-274.

9. Kudina LP, Andreeva RE (2017) F-wave of single firing motor units: correct or misleading criterion of motoneuron excitability in humans? Neurol Sci 38: $465-472$.

10. Koceja DM, Trimble MH, Earles DR (1993) Inhibition of the soleus H-reflex in standing man. Brain research 629: 155-158.

11. Nakazawa K, Miyoshi T, Sekiguchi H, Nozaki D, Akai M, et al. (2004) Effects of loading and unloading of lower limb joints on the soleus H-reflex in standing humans. Clin Neurophysiol 115: 1296-1304.

12. Capaday C, Stein RB (1986) Amplitude modulation of the soleus H-reflex in the human during walking and standing. J Neurosci 6: 1308-1313.

13. Ogawa T, Kim GH, Sekiguchi H, Akai M, Suzuki S, et al. (2009) Enhanced stretch reflex excitability of the soleus muscle in experienced swimmers. Eur J Appl Physiol 105: 199-205.

14. Nielsen J, Crone C, Hultborn H (1993) H-reflexes are smaller in dancers from the Royal Danish Ballet than in well-trained athletes. Eur J Appl Physio Occup Physiol 66: 116-121.

15. Wang Z, Wang S, Shi FY, Guan Y, Wu Y, et al. (2014) The effect of motor imagery with specific implement in expert badminton player. Neuroscience 275: 102-112.

16. Pascual-Leone A, Nguyet D, Cohen LG, Brasil-Neto JP, Cammarota A, et al. (1995) Modulation of muscle responses evoked by transcranial magnetic stimulation during the acquisition of new fine motor skills. J Neurophysio 74: 1037-1045

17. Meugnot A, Agbangla NF, Almecija Y, Toussaint L (2015) Motor imagery practice may compensate for the slowdown of sensorimotor processes induced by short-term upper-limb immobilization. Psychol Res 79: 489-499.

18. Guillot A, Collet C, Nguyen VA, Malouin F, Richards C, et al. (2009) Brain activity during visual versus kinesthetic imagery: an fMRI study. Hum Brain Mapp 30: 2157-2172

19. Lotze M, Halsband U (2006) Motor imagery. J Physiol Paris 99: 386-395.

20. Mizuguchi N, Suezawa M, Kanosue K (2019) Vividness and accuracy: Two independent aspects of motor imagery. Neurosci Res 147: 17-25. 